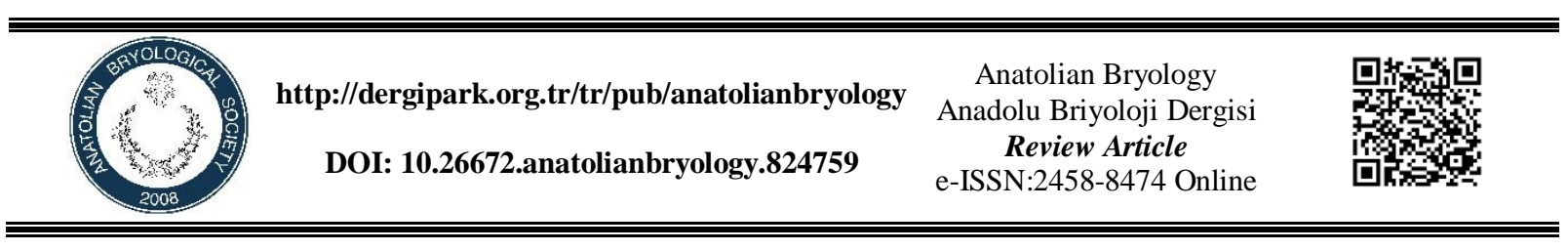

Türkiye'nin B9 Karesi'nin Briyofit Kontrol Listesi

\author{
Mevlüt ALATAŞ ${ }^{1 *} \mathbb{D}$, Serhat URSAVAŞ² ${ }^{2}$ iD \\ ${ }^{1}$ Munzur Üniversitesi, Tunceli Meslek Yüksekokulu, Tunceli, TÜRKIYE \\ ${ }^{2}$ Çankırı Karatekin Üniversitesi, Orman Fakültesi, Orman Mühendisliği Bölümü, Çankırı, TÜRKIYYE
}

\begin{tabular}{lll}
\hline Received: 11 November 2020 & Revised: 14 December 2020 & Accepted: 18 December 2020 \\
\hline
\end{tabular}

$\ddot{O} z$

Bu çalışma, Henderson (1961)'ın Türkiye kareleme sistemine göre B9 karesi içerisinde, şimdiye kadar yapraklı karayosunu (Bryophyta) ve ciğerotları (Marchantiophyta) ile ilgili yapılmış olan araştırmalara dayanan güncel bir listeyi içermektedir. Listede; yapraklı karayosunları için 18 familya ve 54 cinse ait 143 takson, ciğerotları için ise 4 familya ve 5 cinse ait 6 takson olmak üzere toplamda 22 familya, 59 cinse ait 149 tür ve tür altı takson bulunmaktadır. Bu kare içerisinde, içerdiği cins sayısı bakımından en zengin familyalar sırasıyla; Pottiaceae (13), Brachytheciaceae (11), Amblystegiaceae (6), içerdiği takson sayısı açısından ise en zengin familyalar ise; Pottiaceae (43), Brachytheciaceae (21), Grimmiaceae ile Bryaceae (17) ve Orthotrichaceae (12)'dir. Takson çeşitliliği açısından en zengin olanlar cisler; Grimmia (12), Syntrichia (10), Didymodon (9), Ptychostomum ve Tortula (8), Bryum ve Orthotrichum (7)'dir.

Anahtar kelimeler: Karayosunu, Ciğerotu, Kareleme sistemi, B9, Türkiye.

\title{
The Bryophyte Check-List For B9 Square of Turkey
}

\begin{abstract}
This study includes the current list based on moss and liverworts studies done done so far up to now for in B9 grid square of Turkey according to the grid system of Henderson (1961) according to Turkey grid square of Henderson (1961). There are 143 taxa belonging to 18 families and 54 genera for mosses, 6 taxa belonging to 4 families and 5 genera for liverworts, in other words totally 149 taxa (species and subspecies) belonging to 22 families and 59 genera in the list. In this square, the richest families in terms of the number of genera are Pottiaceae (13), Brachytheciaceae (11), Amblystegiaceae (6). Onthe other hand, the richest families in terms of the number of taxa are Pottiaceae (43), Brachytheciaceae (21), Grimmiaceae, Bryaceae (17), and Orthotrichaceae (12). The richest genera in terms of taxa diversity are Grimmia (12), Syntrichia (10), Didymodon (9), Ptychostomum and Tortula (8), Bryum and Orthotrichum (7).
\end{abstract}

Keywords: Moss, Liverwort, Squaring system, B9, Turkey.

\footnotetext{
* Corresponding author: mevlutalatas@hotmail.com

(C) 2021 All rights reserved / Tüm hakları saklıdır.

To cite this article: Alataş M., UrsavaşS S., 2021. Türkiye'nin B9 Karesi'nin Briyofit Kontrol Listesi. Anatolian Bryology. 7:1, 53-59.

(c) (1) () This work is licensed under a Creative Commons Attribution-Non Commercial 4.0 International License.
} 


\section{Giriș}

Türkiye haritası üzerinde yapraklı karayosunları, ciğerotları ve boynuzotları için esas alınan kareleme sistemi 15 kareden oluşmaktadır (Henderson, 1961). Bu grid sistem, Türk briyofitçileri tarafindan kabul görmüş ve son yıllarda yapılan çalışmalarda kullanılmıştır (Özdemir, 2009). Türkiye kareleme sistemine göre
B9 karesi, Türkiye'nin Doğu kesiminde yer almaktadır (38 $\left.40^{\circ} \mathrm{K} ; 38^{\circ} 42^{\circ} \mathrm{D}\right)$ (Sekil 1). Bu kare; Tunceli, Bingöl, Elazığ ve Erzincan illerinin tamamı, Sivas ve Malatya illerinin doğusu, Erzurum ilinin güney batısı, Muş ve Bitlis'in batısı, Diyarbakır ve Adıyamanın kuzeyi ile Siirt ilinin kuzey batısını kapsamaktadır.

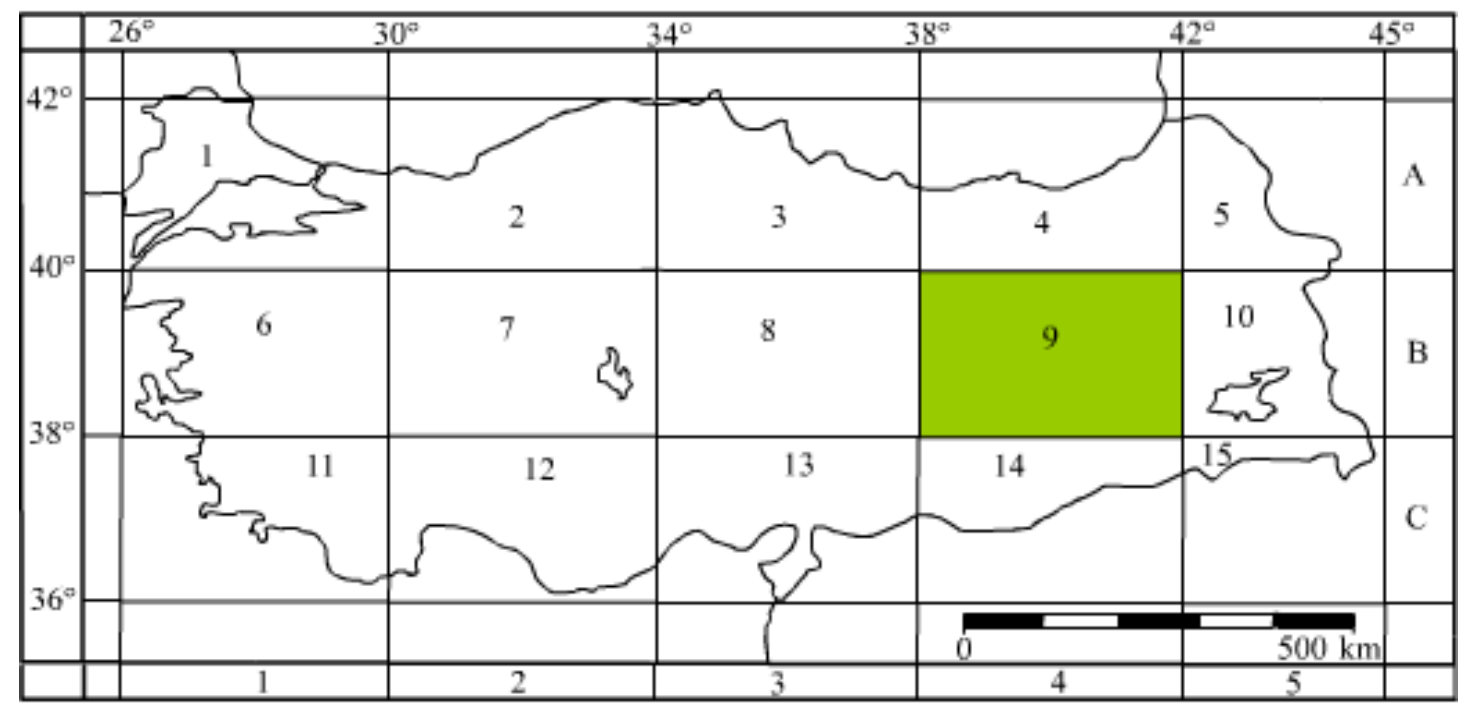

Şekil 1. Türkiye Haritası Kareleme Sistemi (Henderson, 1961)

Ülke geneli için hazırlanan genel briyofit kontrol listeleri [(Çetin, 1988a-1988b), (Uyar ve Çetin, 2004), (Kürschner ve Erdağ, 2005), (Özenoğlu Kiremit ve Keçeli, 2009)] ile birlikte son yıllarda sadece kareler için de briyofit kontrol listeleri hazırlanmıştır. $\mathrm{Bu}$ konuda ilk olarak Özdemir; (2000-2009) A4 karesinde yayılış gösteren briyofit taksonlarını içeren bir liste hazırlamıştır. Daha sonra A2 karesi için Ursavaş ve Abay (2009), A1 karesi için Ursavaş ve ark. (2009), A3 karesi için Abay ve ark. (2009) ve A5 karesi için Şahin ve ark. (2009)'nın bryopsida sinıfi ile ilgili kontrol listelerinin yayımlanmasıyla Türkiye'nin kuzey kesimlerini içeren karelerin (A1-A2-A3-A4-A5) karayosunları listeleri tamamlanmıştır. Yapılan bu çalışmada ise, Türkiye'nin B9 karesinin yapraklı karayosunları ve ciğerotlarını içeren genişletilmiş bir briyofit listesi sunulmuştur.

\section{Materyal ve Metot}

Bu kontrol listesi; Henderson (1961)'un Türkiye kareleme sistemine göre B9 karesi içerisinde yapılmış briyofloristik araştırmaların incelenmesiyle hazırlanmıştır. Şimdiye kadar B9 karesinde briyofitler ile ilgili yapılan çalışmalar sirasiyla; Henderson, 1958; Robinson ve Godrey, 1960; Henderson, 1961-1963; Henderson ve Pentice, 1969; Erdağ ve ark., 2003; Papp, 2007;
Kürschner ve Erdağ, 2009; Erdağ ve Kürschner, 2009a,b; Alataş ve ark., 2014; Alataş ve Batan, 2015, 2016a,b, 2017; Alataş ve Ursavaş, 2019; Alataş ve ark., 2019; Alataş ve ark., 2020'dır. Çalışmalarda tespit edilenlerin yanısıra, B9 karesi içerisine giren diğer ciğerotu taksonlarının tespitinde ise; Özenoğlu Kiremit ve Keçeli (2009)'dan yararlanılmıştır.

$\mathrm{Bu}$ kontrol listesi hazırlanırken Çetin (1988a1988b), Uyar ve Çetin (2004), Kürschner ve Erdağ (2005), Özenoğlu Kiremit ve Keçeli (2009), Erdağ ve Kürschner (2017) gibi Türkiye karayosunlarını ve ciğerotlarını içeren kontol liseleri incelenip, aşağıda isimleri yazılı taksonların ülkemiz bryofit listesinde mevcut olup olmadıkları araştırılmıştır. Bitki listesinin hazırlanışında ciğerotları için Grolle ve Long (2000), karayosunları için Hill ve ark. (2006), geçerli isim ve sinonimlik durumlarının tespitinde ise Ros ve ark., (2013) ve Lara et al. (2016) dikkate alınmıştır. Sistematik kategori ve taksonların alfabetik sıraya göre düzenlenlendiği kontrol listesinin sonunda taksonlara ait sinonimler de sunulmuştur.

\section{Araştırma Sonuçları}

Türkiye haritası kareleme sistemindeki B9 karesinde yapılan briyofloristik araştırmaların 
incelenmesi sonucu ortaya çıkan bu kontrol listesi, son yıllarda yapılan çalışmaların da değerlendirilmesiyle birlikte mevcut durumun tespitinin yanı sıra ülkemizin diğer karelerinde yapılan bu tür çalışmalarla bir karşılaştırma

\section{TAKSON LISTESI}

Marchantiophyta Stotler \& Crand. Stotl.

Jungermanniopsida Stotler \& Crand. Stotl.

Lunulariales H.Klinggr.

Lunulariaceae H.Klinggr.

1 Lunularia Adans.

1 Lunularia cruciata (L.) Dumort. ex Lindb.

Marchantiales Limpr.

Marchantiaceae (Bisch.) Lindl.

2 Marchantia L.

1 polymorpha subsp. polymorpha

2 polymorpha subsp. montivagans Bischl. \& Boisselier.

Metzgeriales Chalaud.

Aneuraceae H.Klinggr.

3 Riccardia Gray.

1 multifida (L.) Gray.

Pelliales He-Nygrén.

Pelliaceae H.Klinggr.

4 Apopellia Grolle.

1 endiviifolia (Dicks.) Nebel \& D.Quandt

5 Pellia Raddi.

1 epiphylla (L.) Corda.

Bryophyta Schimp.

Bryopsida Pax.

Bartramiales D.Quandt, N.E.Bell \& Stech.

Bartramiaceae Schwägr.

1 Philonotis Brid.

1 calcarea (Bruch \& Schimp.) Schimp.

2 fontana (Hedw.) Brid.

Bryales Limpr.

Bryaceae Schwägr.

2 Bryum Hedw.

1 argenteum Hedw.

2 canariense Brid.

3 elegans Nees.

4 funkii Schwägr.

5 gemmiparum De Not.

6 kunzei Hornsch.

7 schleicheri DC.

3 Imbribryum N.Pedersen.

1 alpinum (Huds. ex With.) N.Pedersen.

2 mildeanum (Jur.) J.R.Spence.

4 Ptychostomum Hornsch.

1 capillare (Hedw.) Holyoak \& N. Pedersen.

2 creberrimum (Taylor) J.R. Spence \& H.P.

Ramsay.

3 donianum (Grev.) Holyoak \& N.Pedersen.

4 imbricatulum (Müll. Hal.) Holyoak \& N.

Pedersen.

5 moravicum (Podp.) Ros \& Mazimpaka. olanağını ortaya çıkarmıştır. Bu tür çalışmaların bütün kareler için geçekleştirilmesi durumunda, yapraklı karayosunları, ciğerotları ve boynuzotlarının Türkiye'deki korolojisi ile ilgili çalışmalara önemli kaynak teşkil edecektir.

6 pallens (Sw.) J.R. Spence.

7 pseudotriquetrum (Hedw.) J.R.Spence \& H.P.Ramsay.

8 torquescens (Bruch \& Schimp.) Ros \& Mazimpaka.

Mniaceae Schwägr.

5 Pohlia Hedw.

1 elongata Hedw.

2 wahlenbergii (F.Weber \& D.Mohr)

A.L. Andrews.

3 wahlenbergii (F.Weber \& D.Mohr)

A.L. Andrews var. calcarea (Warnst.)

E.F.Warb.

Dicranales H.Philib. ex M.Fleisch.

Dicranaceae Schimp.

6 Dicranella (Müll.Hal.) Schimp.

1 varia (Hedw.) Schimp.

2 subulata (Hedw.) Schimp.

Fissidentaceae Schimp.

7 Fissidens Hedw.

1 arnoldii R.Ruthe [1]

Pottiaceae Schimp.

8 Anoectangium Schwägr.

1 handelii Schiffn.

9 Barbula Hedw.

1 convoluta Hedw.

2 unguiculata Hedw.

10 Bryoerythrophyllum P.C.Chen. 1 recurvirostrum (Hedw.) P.C.Chen.

11 Cinclidotus P.Beauv.

1 aquaticus (Hedw.) Bruch \& Schimp.

2 riparius (Host ex Brid.) Arn.

3 vardaranus Erdağ \& Kürschner [2]

12 Crossidium Jur.

1 squamiferum (Viv.) Jur. var. squamiferum (Viv.) Jur.

2 var. pottioideum (De Not.) Mönk.

13 Didymodon Hedw.

1 acutus (Brid.) K.Saito.

2 fallax (Hedw.) R.H. Zander.

3 insulanus (De Not.) M.O.Hill.

4 luridus Hornsch.

5 nicholsonii Culm.

6 spadiceus (Mitt.) Limpr.

7 tophaceus (Brid.) Lisa

8 umbrosus (Müll.Hal.) R.H.Zander.

9 vinealis (Brid.) R. H. Zander.

14 Eucladium Bruch \& Schimp. 1 verticillatum (With.) Bruch \& Schimp.

15 Gymnostomum Nees \& Hornsch.

1 aeruginosum $\mathrm{Sm}$.

2 calcareum Nees \& Hornsch. 
3 viridulum Brid.

16 Pterygoneurum Jur.

1 ovatum (Hedw.) Dixon

17 Syntrichia Brid.

1 calcicola J.J.Amann

2 handelii (Schiffn.) S. Agnew \& Vondr.

3 laevipila Brid.

4 latifolia (Bruch ex Hartm.) Huebener.

5 montana Nees.

6 princeps (De Not.) Mitt.

7 ruralis (Hedw.) F.Weber \& D.Mohr.

8 ruralis (Hedw.) F.Weber \& D.Mohr var. ruraliformis (Besch.) Delogne.

9 sinensis (Müll.Hal.) Ochyra.

10 virescens (De Not.) Ochyra.

18 Tortella (Müll.Hal.) Limpr.

1 tortuosa (Hedw.) Limpr.

19 Tortula Hedw.

1 brevissima Schiffn.

2 inermis (Brid.) Mont.

3 muralis Hedw.

4 muralis var. aestiva Brid. ex Hedw.

5 schimperi M.J.Cano, O.Werner \& J.Guerra

6 solmsii (Schimp.) Limpr.

7 subulata Hedw.

8 vahliana (Schultz) Mont.

20 Trichostomum Bruch.

1 brachydontium Bruch.

Encalyptales Dixon.

Encalyptaceae Schimp.

21 Encalypta Hedw.

1 alpina $\mathrm{Sm}$.

2 ciliata Hedw.

3 streptocarpa Hedw.

4 vulgaris Hedw.

Funariales M.Fleisch.

Funariaceae Schwägr.

22 Entosthodon Schwägr.

1 muhlenbergii (Turner) Fife

2 pulchellus (H.Philib.) Brugués

23 Funaria Hedw.

1 hygrometrica Hedw.

Grimmiales M.Fleisch.

Grimmiaceae Arn.

24 Coscinodon Spreng.

1 cribrosus (Hedw.) Spruce.

25 Grimmia Hedw.

1 anodon Bruch \& Schimp.

2 anomala Hampe ex Schimp.

3 funalis (Schwägr.) Bruch \& Schimp.

4 laevigata (Brid.) Brid.

5 montana Bruch \& Schimp.

6 reflexidens Müll.Hal.

7 orbicularis Bruch ex Wilson.

8 ovalis (Hedw.) Lindb.

9 pulvinata (Hedw.) Sm.

10 tergestina Tomm. ex Bruch \& Schimp.

11 trichophylla Grev.
12 unicolor Hook.

26 Schistidium Bruch \& Schimp.

1 apocarpum (Hedw.) Bruch \& Schimp.

2 confertum (Funck) Brunch \& Schimp.

3 helveticum (Schkuhr) Deguchi.

4 platyphyllum (Mitt.) H.Perss.

Hedwigiales Ochyra.

Hedwigiaceae Schimp.

27 Hedwigia P.Beauv.

1 ciliata (Hedw.) P.Beauv.

2 ciliata var. leucophaea Bruch \& Schimp.

Hypnales W.R.Buck \& Vitt.

Amblystegiaceae Kindb.

28 Amblystegium Schimp.

1 serpens (Hedw.) Schimp.

29 Campyliadelphus (Kindb.) R.S.Chopra.

1 chrysophyllus (Brid.) R.S.Chopra.

30 Cratoneuron (Sull.) Spruce.

1 filicinum (Hedw.) Spruce.

31 Hygroamblystegium Loeske.

1 varium (Hedw.) Mönk.

2 varium var. humile (P. Beauv.) Vanderp. \& Hedenäs

3 tenax (Hedw.) Jenn.

32 Leptodictyum (Schimp.) Warnst.

1 riparium (Hedw.) Warnst.

33 Palustriella Ochyra.

1 commutata (Hedw.) Ochyra.

2 falcata (Brid.) Hedenäs

Brachytheciaceae Schimp.

34 Brachytheciastrum Ignatov \& Huttunen.

1 collinum (Schleich. ex Müll.Hal.) Ignatov $\&$ Huttunen.

2 olympicum (Jur.) Vanderp. et al.

3 velutinum (Hedw.) Ignatov \& Huttunen.

35 Brachythecium Schimp.

1 albicans (Hedw.) Schimp.

2 glareosum (Bruch ex Spruce) Schimp.

3 mildeanum (Schimp.) Schimp.

4 rivulare Schimp.

5 rutabulum (Hedw.) Schimp.

36 Homalothecium Schimp.

1 aureum (Spruce) H.Rob.

2 lutescens (Hedw.) H.Rob.

3 philippeanum (Spruce) Schimp.

4 sericeum (Hedw.) Schimp.

37 Kindbergia Ochyra.

1 praelonga (Hedw.) Ochyra.

38 Oxyrrhynchium (Schimp.) Warnst.

1 hians (Hedw.) Loeske.

2 speciosum (Brid.) Warnst.

39 Plasteurhynchium M.Fleisch. ex Broth.

1 striatulum (Spruce) M.Fleisch.

40 Rhynchostegium Schimp.

1 riparioides (Hedw.) Cardot.

41 Rhynchostegiella (Schimp.) Limpr.

1 tenella (Dicks.) Limpr.

42 Rhynchostegium Schimp. 
1 confertum (Dicks.) Schimp.

43 Sciuro-hypnum Hampe.

1 populeum (Hedw.) Ignatov \& Huttunen.

44 Scleropodium Bruch \& Schimp.

1 cespitans (Wilson ex Müll. Hal.) L. F. Koch.

Fontinalaceae Schimp.

45 Fontinalis Hedw.

1 antipyretica Hedw.

Hypnaceae Schimp.

46 Calliergonella Loeske.

1 cuspidata (Hedw.) Loeske.

47 Pylaisia Schimp.

1 polyantha (Hedw.) Schimp.

Leucodontaceae Schimp.

48 Antitrichia Brid.

1 californica Sull.

Pseudoleskeellaceae Ignatov \& Ignatova.

49 Pseudoleskeella Kindb.

1 nervosa (Brid.) Nyholm.

2 rupestris (Berggr.) Hedenäs \& L.Söderstr.

Orthotrichales Dixon.

Orthotrichaceae Arn.

50 Lewinskya F.Lara, Garilleti \& Goffinet.

\section{Ek Açıklamalar}

[1] Türkiye'den ilk kaydı; Fissidens arnoldii R. Ruthe Erdağ \& Kürschner.

[2] Türkiye'den ilk kaydı; Cinclidotus vardaranus Erdağ \& Kürschner.

\section{Sinonimler ve Atılan Takson İsimleri \\ Marchantiophyta \\ Pellia endiviifolia (Dicks.) Dumort. $\rightarrow$ Apopellia endiviifolia (Dicks.) Nebel \& D. Quandt.}

\section{Bryophyta}

Amblystegium riparium (Hedw.) Schimp. $\rightarrow$ Leptodictyum riparium (Hedw.) Warnst.

Brachythecium collinum (Schleich. ex Müll.Hal.) Schimp. $\rightarrow$ Brachytheciastrum collinum (Schleich. ex Müll.Hal.) Ignatov \& Huttunen.

Brachythecium olympicum Jur. Brachytheciastrum Vanderp. et al.

Bryum caespiticium Hedw. $\rightarrow$ Ptychostomum imbricatulum (Müll. Hal.) Holyoak \& N. Pedersen.

Bryum alpinum Huds. ex With. $\rightarrow$ Imbribryum alpinum (Huds. ex With.) N. Pedersen.

Bryum capillare Hedw. $\rightarrow$ Ptychostomum capillare (Hedw.) Holyoak \& N. Pedersen.

Bryum creberrimum Taylor. $\rightarrow$ Ptychostomum creberrimum (Taylor) J.R. Spence \& H.P. Ramsay.

Bryum donianum Grev. $\rightarrow$ Ptychostomum donianum (Grev.) Holyoak \& N. Pedersen.
1 affinis (Schrad. ex Brid.) F.Lara, Garilleti \& Goffinet.

2 rupestris (Schleich. ex Schwägr.) F.Lara, Garilleti \& Goffinet.

3 striata (Hedw.) F.Lara, Garilleti \& Goffinet.

4 speciosa (Nees) F.Lara, Garilleti \& Goffinet.

51 Orthotrichum Hedw.

1 anomalum Hedw.

2 cupulatum Brid.

3 var. cupulatum Brid. var. riparium Huebener.

4 var. cupulatum var. bistratosum Schiffn.

5 pallens Bruch ex Brid.

6 pumilum Sw. ex anon.

7 sprucei Mont.

52 Zygodon Hook. \& Taylor.

1 rupestris Schimp. ex Lorentz.

Splachnales Ochyra.

Splachnaceae Grev. \& Arn.

53 Tayloria Hook.

1 lingulata (Dicks.) Lindb.

Bryum mildeanum Jur. $\rightarrow$ Imbribryum mildeanum (Jur.) J.R. Spence.

Bryum moravicum Podp. $\rightarrow$ Ptychostomum moravicum (Podp.) Ros \& Mazimpaka.

Bryum pallens Sw. $\rightarrow$ Ptychostomum pallens (Sw.) J.R. Spence.

Bryum pseudotriquetrum (Hedw.) P. Gaertn. et al. $\rightarrow$ Ptychostomum pseudotriquetrum (Hedw.) J.R. Spence \& H.P. Ramsay.

Bryum torquescens Bruch \& Schimp. $\rightarrow$ Ptychostomum torquescens (Bruch \& Schimp.) Ros \& Mazimpaka.

Eurhynchium praelongum (Hedw.) Schimp. $\rightarrow$ Kindbergia praelonga (Hedw.) Ochyra.

Funaria mediterranea Lindb. $\rightarrow$ Entosthodon muhlenbergii (Turner) Fife.

Funaria pulchella $\mathrm{H}$. Philib. $\rightarrow$ Entosthodon pulchellus (H. Philib.) Brugués.

Hygroamblystegium humile (P. Beauv.) Vanderp., Goffinet \& Hedenas. $\rightarrow$ Hygroamblystegium varium var. humile (P. Beauv.) Vanderp. \& Hedenäs.

Orthotrichum affine Schrad. Ex Brid. $\rightarrow$ Lewinskya affinis (Schrad. ex Brid.) F. Lara, Garilleti \& Goffinet.

Orthotrichum cupulatum Hoffm. ex Brid. $\rightarrow$ Orthotrichum cupulatum Brid.

Orthotrichum cupulatum Hoffm. ex Brid. var. riparium Huebener. $\rightarrow$ Orthotrichum cupulatum Brid. var. riparium Huebener.

Orthotrichum rupestre Schleich. ex Schwägr. $\rightarrow$ Lewinskya rupestris (Schleich. ex Schwägr.) F. Lara, Garilleti \& Goffinet. 
Orthotrichum speciosum Nees. $\rightarrow$ Lewinskya speciosa (Nees) F. Lara, Garilleti \& Goffinet.

Orthotrichum striatum Hedw. $\rightarrow$ Lewinskya striata (Hedw.) F. Lara, Garilleti \& Goffinet.

Platyhypnidium riparioides (Hedw.) Dixon $\rightarrow$ Rhynchostegium riparioides (Hedw.) Cardot.

Schistidium rivulare (Brid.) Podp. $\rightarrow$ Schistidium helveticum (Schkuhr) Deguchi.

Not: $\mathrm{Bu}$ çalışma, 21. Ulusal Biyoloji Kongresi (03-07 Eylül 2012, İzmir)'nde poster bildiri olarak sunulmuştur.

\section{Kaynaklar}

Abay G. Ursavaș S. Şahin A. 2009. Türkiye'nin A3 karesinin karayosunları (Musci) kontrol listesi. I. Ulusal Batı Karadeniz Ormancılık Kongresi Bildiriler Kitabı (Özel sayı). 2, 613-619.

Alataş M. Batan N. Hazer Y. 2014. The Moss Flora of Elazı̆g-Sivrice (Turkey) Province. Biological Diversity and Conservation. 7:2, 148-153.

Alataş M. Batan N. 2015. The Moss Flora of Keban (Elazı ğ/Turkey) District. Biological Diversity and Conservation. 8:2, 59-65.

Alataş M. Batan N. 2016a. The Moss Flora of Arapgir (Malatya/Turkey) District. Biological Diversity and Conservation. 9:2, 102-107.

Alataş M. Batan N. 2016b. The Moss Flora of Yeşilyurt and Battalgazi (Malatya/Turkey) District. Anatolian Bryology. 2 :1-2, 47-55.

Alataş M. Batan N. 2017. The bryophyte flora of Baskil district (Elazı̆̆/Turkey). Biological Diversity and Conservation. 10:1, 31-38.

Alataş M. Ursavaş S. 2019. The bryophyte flora of Palu (Elazığ /Turkey) district, Biological Diversity and Conservation. 12:1, 81-88.

Alataş M. Batan N. Erata H. 2019. The Moss Flora of Pertek (Tunceli /Turkey) District. Biological Diversity and Conservation. 12:2, 23-30.

Alataş M. Batan N. Erata H. Özen Ö. 2020. The Moss Flora of Bingöl Central District (Turkey). Anatolian Bryology. 6:1, 55-63.

Çetin B. 1988a. Checklist of Mosses of Turkey. Lindbergia. 14, 15-23.

Çetin B. 1988b. Checklist of the liverworts and hornworts of Turkey. Lindbergia. 14, 1214.

Erdağ A. Kürschner H. 2009a. Cinclidotus vardaranus Erdağ \& Kürschner (Bryopsida, Pottiaceae sp. nov. from Estern Turkey, with some remarks on the speciation centre of the genus. Nova Hedwigia. 88:1-2, 183188.
Erdağ A. Kürschner H. 2009b. Fissidens arnoldii R. Ruthe. New national and regional bryophyte records, 20. Journal of Bryology. 31, 54-62.

Erdağ A. Kırmac1 M. Kürschner H. 2003. The Hedwigia ciliata (Hedw.) Ehr. ex P.Beauv. Complex in Turkey, with a New Record, $\mathrm{H}$. ciliata var. leucophaea Bruch \& Schimp. (Hedwigiaceae, Bryopsida). Turkish Journal of Botany. 27, 349-356.

Erdağ A. Kürschner H. 2017. Türkiye Bitkilerinin Listesi (Karayosunları). Ali Nihat Gokyiğit Vakfi Bas. İstanbul.

Grolle R. Long D.G. 2000. An annotated check-list of the Hepaticeae and Anthocerotae of European and Macaronesia. Journal of Bryology. 22, 103-140.

Henderson D.M. 1958. Contributions to the Bryophyte Flora of Turkey III. Notes from the Royal Botanic Garden Edinburgh. 22, 611-620.

Henderson D.M. 1961. Contributions to the bryophyte flora of Turkey IV: Notes from the Royal Botanic Garden Edinburgh. 23, 263-278.

Henderson D.M. 1963. Contributions to the bryophyte flora of Turkey VI. Notes from the Royal Botanic Garden Edinburgh. 25, 279-291.

Henderson D.M. Pentice H.T. 1969. Contributions to the Bryophyte Flora of Turkey VIII. Notes from the Royal Botanic Garden Edinburgh. 29, 235-262.

Henderson D.M. Prentice H.D. 1969. Contributions to the bryophyte flora of Turkey: VIII. Notes from the Royal Botanic Garden Edinburgh. 29, 235-262.

Hill MO. Bell N. Buruggeman-Nannenga MA. Brugues M. Cano MJ. vd. 2006. An annotated checklist of the mosses of Europe and Macronesia. Journal of Bryology. 28, 198267.

Kürschner H. Erdağ A. 2005. Bryophytes of Turkey: An Annotated Reference List of the Species with Synonymus from the Recent Literature and an Annotated List of Turkish Bryological Literature. Turkish Journal of Botany. 29, 95-154.

Kürschner H. Erdağ A. 2009. The Grimmietum commutato-campestris in Turkey. Ecology and life Syndromes of a saxicolous bryophyte community with the description of two new subassociations. Nova Hedwigia. 88: 3-4, 441-463.

Lara F. Garilleti R. Goffinet B. Draper I. Medina R. Vigalondo B. Mazimpaka V. 2016. Lewinskya, a new genus to accommodate the phaneroporous and monoicous taxa of 
Orthotrichum (Bryophyta, Orthotrichaceae). Cryptogamie, Bryol. 37, 361-382.

Özdemir T. 2000. Checklist of the Bryophyta of A4 square of Turkey. Energy, Education, Science \& Technology. 4: 2, 60-79.

Özdemir T. 2009. A Revised check-list of the Bryophytes of A4 Square of Turkey. International Journal of Botany. 5:1, 1-35.

Özenoğlu Kremit H. Keçeli T. 2009. An Annotated Check-list of the Hepaticae and Anthocerotae of Turkey. Cryptogamie, Bryologie. 30:3, 343-356.

Papp B. 2007. Contributions to the Bryophyte Flora of Eastern Turkey. Studia Botanica Hungaria. 38, 71-78.

Robinson H. Godrey R.K. 1960. Controbutions to the bryophyte flora of Turkey. Revue Bryologique et Lichènologique. 29, 244253.

Ros R.M. Mazimpaka V. Abou-Salama U. Aleffi M. Blockeel T.L. Brugués vd. 2013. Mosses of the Mediterranean, an annotated checklist. Cryptogamie, Bryol. 34, 99-283.

Şahin A. Ursavaş S. Abay G. 2009. Türkiye'nin A5 karesinin karayosunları (Musci) kontrol listesi. I. Ulusal Batı Karadeniz Ormancılık Kongresi Bildiriler Kitabı (Özel sayı). 2, 620-625.

Ursavaş S. Abay G. 2009. Türkiyenin A2 karesinin karayosunları (Musci) kontrol listesi. Bartın Orman Fakültesi Dergisi. 16:2, 33-43.

Ursavaş, S. Şahin A. Abay G. 2009. Türkiye'nin A1 karesinin karayosunları (Musci) kontrol listesi. I. Ulusal Batı Karadeniz Ormancılık Kongresi Bildiriler Kitabı (Özel sayı). 2, 604-612.

Uyar G. Çetin B. 2004. A new check-list of the mosses of Turkey. Journal of Bryology. 26, 203-220. 